\title{
Philosophiques
}

\section{Justice et politiques linguistiques : pourquoi les laisser-fairistes devraient exiger des interventions de l'État}

\section{David Robichaud}

Volume 38, numéro 2, automne 2011

URI : https://id.erudit.org/iderudit/1007458ar

DOI : https://doi.org/10.7202/1007458ar

Aller au sommaire du numéro

Éditeur(s)

Société de philosophie du Québec

ISSN

0316-2923 (imprimé)

1492-1391 (numérique)

Découvrir la revue

Citer cet article

Robichaud, D. (2011). Justice et politiques linguistiques : pourquoi les laisser-fairistes devraient exiger des interventions de l'État. Philosophiques, 38(2), 419-438. https://doi.org/10.7202/1007458ar
Résumé de l'article

La neutralité bienveillante est parfois considérée comme la seule politique linguistique compatible avec l'impératif de neutralité de l'État libéral. Un argument en faveur d'une telle position est celui de la coïncidence entre l'équilibre des choix individuels et l'optimalité des résultats. Nous démontrons que la présence d'échecs de marché (externalités et asymétrie d'informations) empêche cette coïncidence sur le marché linguistique et que des interventions étatiques, dont la nature demeure à préciser, sont alors nécessaires pour rétablir cette coïncidence. 


\title{
Justice et politiques linguistiques: pourquoi les laisser-fairistes devraient exiger des interventions de l'État
}

\author{
DAVID ROBICHAUD
}

Université d'Ottawa

\begin{abstract}
RÉSUMÉ. - La neutralité bienveillante est parfois considérée comme la seule politique linguistique compatible avec l'impératif de neutralité de l'État libéral. Un argument en faveur d'une telle position est celui de la coïncidence entre l'équilibre des choix individuels et l'optimalité des résultats. Nous démontrons que la présence d'échecs de marché (externalités et asymétrie d'informations) empêche cette coïncidence sur le marché linguistique et que des interventions étatiques, dont la nature demeure à préciser, sont alors nécessaires pour rétablir cette coïncidence.
\end{abstract}

\begin{abstract}
Benign neglect is sometimes considered the only language policy available for a neutral liberal state. An argument supporting this position is the coincidence of choices equilibrium and results optimality. We show that market failures (externalities and asymmetrical information) on the linguistic market breaks this coincidence and therefore that some state interventions are legitimate even from a laisser-faire framework.
\end{abstract}

MOTS-CLÉS: Politiques linguistiques, neutralité bienveillante, échecs de marché, marché linguistique

KEYWORDS: Language policies, benign neglect, market failures, language market

\section{Introduction}

L'État libéral peut-il se permettre d'intervenir dans les décisions culturelles et linguistiques de ses citoyens? Le fait que certaines communautés culturelles et linguistiques aient du mal à rivaliser avec des communautés dominantes représente-t-il un problème moral auquel l'État doit s'attaquer, ou s'agit-il plutôt d'un phénomène que l'on peut déplorer mais que l'on ne doit pas tenter d'enrayer? Les auteurs libéraux s'opposent sur cette question, certains défendant la nécessité pour l'État de se limiter à une attitude de neutralité bienveillante ou de douce négligence (bening neglect), d'autres justifiant la légitimité de l'État d'intervenir afin de venir en aide à certaines communautés minoritaires. Notre objectif dans cet article est de prendre position en faveur du second camp, ceux que l'on appelle parfois les "culturalistes libéraux ", en démontrant que la justification morale la plus forte de la neutralité bienveillante pose plusieurs problèmes. Nous irons plus loin en tentant de démontrer que nous pouvons justifier des interventions de l'État sur la base même des arguments pouvant être employés par les défenseurs de la neutralité bienveillante. Dans un passage célèbre de La citoyenneté multi- 
culturelle (200I), Kymlicka a déjà argumenté de façon convaincante qu'un État ne peut pas être neutre d'un point de vue linguistique. L'État et ses employés devront nécessairement s'adresser à la population dans une ou plusieurs langues, mais il n'est pas possible dans l'état actuel des choses d'imaginer que l'État puisse être neutre dans ses rapports linguistiques. Nous emboîterons le pas à cet argument en allant plus loin. Nous n'avançons pas simplement que la neutralité bienveillante est inaccessible en raison de l'impossibilité pour l'État d'être parfaitement neutre en matière linguistique. Nous avançons que, même si l'État pouvait être neutre, il ne devrait pas l'être puisque son intervention est nécessaire afin de réaliser les promesses des politiques de laisser-faire: respecter la liberté des individus et permettre la production de résultats collectifs aussi souhaitables que possible.

Les défenseurs de la neutralité bienveillante considèrent que l'État libéral doit "laisser faire» les individus dans les domaines linguistiques et culturels. Le rôle de l'État libéral devrait selon eux se limiter à protéger la liberté négative des citoyens en garantissant que ni l'État ni d'autres citoyens ne pourront y faire intrusion. Le paysage linguistique sera ainsi construit par des décisions individuelles, mais sans être déterminé par un dessein politique ou moral. Les principales critiques de l'adoption de politiques « laisser-fairistes » ou de "neutralité bienveillante» mettent en lumière le fait que les contacts linguistiques se font aux détriments des langues dominées et que le refus d'intervenir pour protéger les langues minoritaires ou dominées revient à accepter l'assimilation de plusieurs minorités (Laponce, I984; Coulombe, I995: 93). Cette critique est tout à fait fondée. Le problème est que le clan laisser-fairiste, davantage préoccupé par le processus produisant des résultats collectifs que par les résultats eux-mêmes, peut très bien répondre: «Et alors?». Pour que l'on puisse voir un problème moral à la disparition des langues et des cultures, on doit défendre soit la valeur intrinsèque des langues ou de la diversité linguistique (Réaume, 2000), soit démontrer que les locuteurs minoritaires sont victimes d'injustices et ne disposent pas des mêmes possibilités de choix que les locuteurs majoritaires (Kymlicka, 200I). $\mathrm{Si}$ on rejette l'idée d'une valeur intrinsèque des langues ou de la diversité linguistique, qui pose d'importants problèmes conceptuels et justifie des politiques linguistiques contre-intuitives (Weinstock, 2003a), on ne peut s'opposer au laisser-faire en raison du résultat vers lequel il précipite le paysage linguistique. On peut trouver regrettable que des langues disparaissent et que des individus voient disparaître leur langue maternelle, mais on ne saurait justifier sur cette base des interventions de l'État visant à contraindre les comportements individuels. C'est plutôt la seconde voie que nous explorerons dans cet article.

Si les politiques linguistiques de laisser-faire ne sont pas souhaitables, ce n'est pas parce qu'elles ne font rien pour protéger ou promouvoir la diversité linguistique. C'est plutôt parce qu'elles présupposent un contexte d'interactions entre les individus que l'on ne rencontre pas dans la réalité. 
Ce que nous entendons démontrer, c'est que le laisser-faire n'est pas plus prometteur sur le marché linguistique qu'il ne l'est sur le marché économique, et ce pour les mêmes raisons: la présence d'échecs de marché. Le libre marché linguistique, pour remplir ses promesses, aura besoin d'interventions de l'État afin de corriger ces échecs de marché. Si les économistes sont conscients de ces interventions nécessaires dans la sphère économique, il est grand temps que les spécialistes des questions linguistiques en prennent conscience à leur tour dans leurs commentaires sur la situation linguistique. Nous allons d'abord présenter l'argument laisser-fairiste qui représente, à notre avis, l'argument le plus solide en faveur de la neutralité bienveillante. Nous allons ensuite présenter les conditions que le marché doit remplir pour que l'on puisse laisser les acteurs agir librement et espérer obtenir des résultats optimaux. Nous terminerons en présentant différents échecs de marché qu'on ne peut manquer de rencontrer dans la sphère linguistique et qui justifient le rejet du laisser-faire linguistique. Les échecs de marché présentés découlent principalement du multilinguisme qui est maintenant une quasi nécessité et qui complexifie les choix linguistiques faits par les individus. Les individus manifestent plusieurs préférences par l'apprentissage, l'usage et la transmission des langues, et c'est la tentative de satisfaire ces préférences simultanément sans stratégie collective qui cause des échecs de marché et des problèmes d'action collective. L'identification de ces échecs de marché ne permet pas seulement de rejeter le laisser-faire et la neutralité bienveillante en matière linguistique. Il nous sera par la suite possible de nous orienter dans l'élaboration de politiques linguistiques qui seront justifiées par les arguments mêmes du laisser-faire: le respect de la liberté individuelle et la production de résultats aussi souhaitables que possible.

\section{Le laisser-faire comme politique linguistique}

Le laisser-faire est parfois présenté comme la seule politique linguistique à la portée d'un État libéral désireux d'éviter de limiter arbitrairement la liberté de ses citoyens, de s'ingérer de manière paternaliste dans leurs vies et de favoriser certaines conceptions du bien (voir Kukathas, I998). L'État devrait se limiter à faire respecter la sphère de liberté négative de chaque citoyen et s'assurer que personne n'est forcé ou empêché de parler la langue de son choix dans différents contextes privés. L'égalité est assurée par l'égalité de droits indépendamment de l'identité des individus (Rawls, I975; Kukathas, I992). Toutefois, la liberté linguistique n'implique pas de protections linguistiques de la part de l'État ni de devoirs d'autrui à nous répondre ou même à nous comprendre dans cette langue. C'est à chacun de s'adapter à son contexte et à faire les choix qui sont à son avantage. Intervenir pour favoriser certaines communautés linguistiques reviendrait à défavoriser des individus non membres de ces communautés. Or si on peut justifier l'intervention de l'État en se basant sur l'importance des langues et des cultures pour les individus, on peut aussi justifier sa non-intervention sur la même 
base. L'intervention de l'État n'élimine pas les coûts que doivent assumer les locuteurs minoritaires, elle les transfère sur les locuteurs qui ne sont pas privilégiés par cette intervention. Si la langue est importante pour les uns, il est difficile de justifier pourquoi elle le serait moins pour les autres, et il est difficile de justifier ce transfert de coûts. Or si le choix de la langue d'usage a la même importance pour tout individu, peu importe le statut de la communauté dont il fait partie, on voit mal comment on peut modifier au moyen d'institutions les coûts et bénéfices accompagnant la situation linguistique «naturelle» produite par des interactions libres.

Les fondements normatifs de la position laisser-fairiste sont solides et méritent d'être mis en lumière. Les vertus du laisser-faire ont été identifiées de façon limpide par les sciences économiques. Celles-ci ne se limitent pas à exiger l'absence de contraintes aux libertés individuelles sans considération des résultats. Il est vrai que le marché est présenté comme un mécanisme naturel de coordination des préférences individuelles qui respecte davantage la liberté et la diversité humaine que ne le font des mécanismes de coordination politiques (Friedman, 20I0: 68). Mais le marché est aussi considéré comme étant le meilleur moyen de coordonner les projets individuels et de produire des résultats collectivement optimaux. On pense que la meilleure façon d'obtenir des résultats collectivement désirables est de laisser chaque agent libre d'agir comme bon lui semble tant qu'il demeure dans les limites de la justice, c'est à dire tant qu'il respecte les libertés d'autrui et assume les coûts de ses actions. Comme chaque individu est le mieux placé pour décider de ce qui est bon et a de la valeur pour lui, laisser chacun maximiser rationnellement et librement son utilité est la meilleure façon d'obtenir des résultats collectivement optimaux.

L'idée géniale de la main invisible est que les intérêts individuels et l'intérêt collectif sont servis simultanément par les initiatives individuelles, en d'autres termes, que l'équilibre coïncide avec l'optimal. Un choix est en équilibre lorsque celui-ci offre un résultat qui maximise l'utilité de l'agent compte tenu du contexte et des comportements d'autrui. Ce contexte peut ne pas lui permettre de satisfaire sa préférence dominante, son premier choix, par exemple de maintenir sa langue ancestrale et de vivre dans un contexte linguistique où cette langue est dominante. (C'est ce point qui pose problème pour plusieurs critiques, comme nous l'avons mentionné.) Son choix sera néanmoins pour lui aussi bénéfique que possible, compte tenu des circonstances et des choix des autres agents. Un résultat est quant à lui optimal si aucun autre résultat ne lui est préféré par tous les agents. Les interventions de l'État ne sont légitimes que lorsque les comportements libres de chacun produisent des résultats sub-optimaux, c'est-à-dire que les choix en équilibre ne produisent plus des résultats optimaux. L'intervention est alors justifiée parce que chacun des agents reconnaît que celle-ci est nécessaire pour leur permettre de satisfaire leurs préférences individuelles de la façon la plus efficace possible. 
Les mécanismes du marché permettent donc de réconcilier les intérêts individuels et l'intérêt collectif, ou l'équilibre et l'optimalité, mais à certaines conditions. Le marché idéal présuppose entre autres conditions la rationalité parfaite des agents et l'indépendance des fonctions d'utilité. Cela implique que les actions posées par chacun ne seront orientées que vers la maximisation de l'utilité de l'agent, et que ces actions n'influenceront ni à la hausse ni à la baisse l'utilité dont bénéficie autrui. Dans une telle situation, il n'y a pas de prise pour la morale ou pour l'intervention de l'État (Gauthier 2000: I23-I 55). Il existe un équilibre naturel que toute intervention exogène ne pourrait que bousculer. Chaque individu profite au maximum de ses ressources, sans nuire ni subir la nuisance de quiconque, et chacun n'a que lui à blâmer si sa situation est moins enviable que celle d'autrui. Toute intervention de l'État, toute taxation ou redistribution de ressources pourra dissuader certains de produire des biens qui auraient été désirables par plusieurs ou par tous, et ainsi briser la coïncidence entre l'équilibre des choix et l'optimalité des résultats. Dans le même ordre d'idées, on pourrait avancer que toute restriction aux choix linguistiques des individus nuirait à l'optimalité linguistique (et économique). C'est sur cette base que l'on peut justifier une politique de laisser-faire. Puisque l'intervention de l'État n'améliore pas le résultat collectif mais qu'il contraint certains individus au nom de principes ou de valeurs qu'ils peuvent ne pas accepter ou valoriser, il est plus efficace de laisser faire les individus.

Il subsiste cependant un obstacle de taille sur la route menant de l'équilibre à l'optimalité. Pour que l'on puisse se passer de l'État (ou d'autres interventions exogènes), et profiter des bénéfices offerts par des interactions libres, on doit pouvoir compter sur un "marché concurrentiel idéal». "Mais cela n'est pas possible» (Gauthier, 2000: I24). Hors de ce marché concurrentiel parfait, il n'y a pas coïncidence de l'équilibre et de l'optimalité. Les individus désireux de maximiser leur utilité et de satisfaire leurs préférences produiront malgré eux des résultats collectivement suboptimaux ou carrément désastreux. Sans la main invisible pour coordonner les projets individuels et les diriger vers un optimal collectif, nous aurons besoin de la «main visible» de l'État. Chaque individu appellera de ses vœux cette intervention de l'État qui sera jugée rationnellement nécessaire pour permettre de corriger les échecs de marché. L'identification d'échecs de marché permet ainsi d'une part de justifier des interventions exogènes à celui-ci, mais aussi de cibler précisément, ce qui nous permettra d'une part de rejeter le laisser-faire linguistique comme politique optimale, mais aussi d'identifier des moyens de corriger ces échecs de marché qui empêchent le laisser-faire de remplir ses promesses.

\section{Rejet du laisser-faire linguistique}

On peut identifier principalement six types d'échecs de marché qui empêchent l'émergence d'un marché linguistique idéal, ou d'une situation où l'équilibre des choix individuels produit des résultats optimaux (Grin, 2006: 84). 
I) Lorsque les informations dont disposent les agents sont insuffisantes pour qu'ils puissent prendre des décisions rationnelles;

2) Lorsque des coûts de transaction empêchent certaines ententes d'être conclues;

3) Lorsqu'il n'existe pas de marché pour certains biens;

4) Lorsqu'il existe des imperfections de marché telles que des oligopoles ou des monopoles qui troublent l'équilibre entre l'offre et la demande;

5) Lorsqu'il y a présence d'externalités, qu'elles soient positives ou négatives;

6) Lorsqu'il existe des biens publics.

Ce que l'on doit noter, c'est que la présence d'un seul de ces types d'échecs de marché détruit l'harmonie entre l'intérêt commun et les intérêts individuels poursuivis par des stratégies individuelles. Lorsque nous prenons une décision quant à la langue à utiliser dans un contexte donné, la langue à apprendre soi-même ou à apprendre à nos enfants, la langue dans laquelle produire des travaux scientifiques ou des produits culturels, nous tentons de réaliser une situation qui est désirable. Si chacun réalise la meilleure situation pour lui-même dans un contexte fixe, on pourrait s'attendre à ce que le résultat collectif de l'ensemble des choix collectifs faits par les locuteurs d'une région soit optimal, c'est-à-dire qu'il ne puisse être amélioré sans aller à l'encontre de la volonté de certains locuteurs. Le problème, c'est que dès qu'une condition n'est pas remplie et qu'il existe un échec de marché, des interventions exogènes sont nécessaires pour rétablir la coïncidence entre équilibre et optimal, et il est faux de penser qu'un marché "presque idéal» peut être corrigé par une intervention étatique «presque nulle». La présence du moindre échec de marché peut exiger des interventions institutionnelles importantes afin de rétablir la coïncidence entre l'équilibre et l'optimalité (Lipsey \& Lancaster I956-I957). Il est important de mentionner qu'il n'est pas garanti que de telles interventions exogènes produiront ou nous rapprocheront d'une situation optimale. Elles s'avèrent néanmoins nécessaires pour tenter de rétablir la coïncidence entre l'équilibre et l'optimalité, et c'est sur cette base que nous les justifierons. Nous démontrerons rapidement que l'on ne peut laisser les choix linguistiques aux forces du marché et aux libres choix individuels puisque les langues sont au cœur de problèmes relevant de ces six types d'échecs de marché. Faute d'espace, nous nous concentrerons sur deux types d'échecs de marché qui sont particulièrement importants sur le marché linguistique: le problème de l'asymétrie d'informations et le problème des externalités. Les autres types d'échecs de marché ont été traités ailleurs. Les problèmes de coûts de transaction (Robichaud et De Schutter, à paraître; Barro, I996; Grin, 2006), d'absence de certains marchés (l'absence des générations futures sur les marchés notamment) (Robichaud et Turmel, 20I0), la présence de monopole (Carr, I985), et la définition des langues comme biens publics ou comme biens hypercollectifs (De Swaan, 2005; Grin, 2006) ont été soulevés et traités ailleurs et auraient pu être repris ici si l'espace nous l'avait permis. 


\section{Asymétrie d'informations ou informations incomplètes}

Tout d'abord, le marché idéal présuppose que les agents disposent de toute information pertinente pour former leurs préférences et faire leurs choix quant aux meilleures façons de les satisfaire. Cette information pertinente, dans le cas qui nous intéresse, inclut les préférences linguistiques d'autrui. Sur le marché économique, un intermédiaire entre les agents permet assez précisément d'identifier les préférences ainsi que l'intensité de celles-ci: le système de prix. Plus des individus désirent intensément et plus ils sont nombreux à désirer un bien, plus son prix sera élevé. Or les langues étant des biens collectifs, une augmentation de la demande ne fera pas en sorte que son prix ou son coût d'apprentissage augmentera. Au contraire. Cependant, en raison des externalités de réseaux, une augmentation du nombre d'individus l'apprenant modifiera à la hausse la valeur qu'aura cette langue pour un individu. Les coûts d'apprentissage en seront réduits, les occasions de l'utiliser seront quant à elles multipliées. Dès lors, connaître les préférences et les intentions d'autres locuteurs faisant partie de notre communauté peut s'avérer nécessaire pour faire des choix linguistiques rationnels. Si nous ne disposons pas d'informations parfaites en matière de préférences linguistiques des individus qui nous entourent, nous pouvons être assurés que nos choix individuels ne produiront pas des résultats collectifs optimaux et que les meilleures stratégies individuelles ne produiront pas les résultats souhaités pour plusieurs individus.

\section{La présence d'externalités sur un marché}

Ensuite, on retrouve le problème le plus intéressant et le plus important pour les langues: la présence d'externalités. La théorie économique accorde une très grande importance aux externalités, positives ou négatives, puisque ces dernières se doivent d'être internalisées pour que le marché idéal soit réalisé. Une externalité est une conséquence d'un acte de production d'échange ou de consommation qui n'affecte pas ou marginalement l'utilité de celui qui pose l'action, mais qui affecte l'utilité d'agents qui ne sont pas parties prenantes de la transaction ou de l'action, ou qui ne le sont pas volontairement. La présence d'externalités implique donc qu'il n'existe pas d'indépendance des fonctions d'utilité. Ce sont des coûts et des bénéfices «externes» en ce sens qu'ils ne sont pas pris en considération dans le calcul d'utilité qui détermine l'action des individus. Ils sont externalisés, c'est-à-dire qu'ils sortent du calcul d'utilité de l'agent, mais ils demeurent bien réels et devront être assumés par quelqu'un. Tenant pour acquis que les individus agissent de façon à maximiser leur utilité, un individu calculera les coûts et les bénéfices d'une action avant de la poser et n'agira que s'il s'agit de l'action qui produit le plus de bénéfices pour lui. Les bénéfices profitant à autrui (externalités positives) et les coûts imposés à autrui (externalités négatives) ne feront pas partie de son calcul de coûts bénéfices. Le problème des externalités, c'est qu'elles font que les coûts privés et les coûts sociaux ou collectifs ne sont pas 
identiques. En d'autres mots, elles troublent la coïncidence de l'équilibre des choix individuels et de l'atteinte de résultats optimaux. Certaines actions produisant des externalités négatives seront désirables pour les individus, puisqu'ils n'en assument pas tous les coûts, mais collectivement indésirables. À l'opposé, certaines actions produisant des externalités positives seront individuellement indésirables mais collectivement désirables. Utiliser sa voiture est très avantageux pour les individus, en partie en raison de l'externalisation des coûts de pollution atmosphérique et sonore ainsi que des risques accrus de collisions mortelles. À l'inverse, éclairer la partie de la rue dans laquelle on habite est peu avantageux puisqu'on est seuls à en assumer les coûts et que cela n'améliore que très marginalement notre sécurité personnelle. Pour que les actions individuelles libres produisent un résultat collectif optimal, on doit donc internaliser les externalités, c'est-à-dire faire en sorte que ceux qui créent des coûts les assument et que ceux qui profitent des bénéfices offrent des compensations.

Ce problème d'inefficience économique découlant des externalités peut aussi prendre la forme d'un problème moral. D'un point de vue économique, ce qui pose problème avec les externalités négatives, c'est que la recherche de la maximisation individuelle ne nous amène pas vers l'optimum collectif promis et attendu. D'un point de vue moral, le problème a trait à l'imposition unilatérale de coûts à des individus ou des communautés n'ayant pas donné leur accord à la production de ces coûts. Donc, d'un côté, le problème est l'absence d'un marché permettant d'internaliser ces externalités, de l'autre, le problème est l'imposition unilatérale de coûts à autrui. On ne peut accepter moralement que des agents imposent une partie des coûts de la satisfaction de leurs préférences à autrui sans son consentement.

En outre, les biens collectifs ayant des structures de réseaux, tels que tous les instruments de communication, y compris les langues, font naître des externalités d'un type particulier auquel nous avons fait référence plus haut: les externalités de réseaux. Ces externalités d'un type particulier ont été identifiées par plusieurs commentateurs et ont permis la justification de certaines interventions étatiques dans la sphère linguistique. (Dalmazzone, I999; Church \& King, I993). Ce sont des externalités positives d'un genre particulier qui sont propres à tout type de bien de consommation ou de production ayant une structure de réseau. L'utilité de chaque personne qui fait partie d'un réseau est modifiée par les actions de quiconque se joint ou se retire du réseau. Les bénéfices que l'on retire d'avoir un téléphone, un lecteur Blu-Ray, un Mac, sont fonction du nombre de personnes qui ont fait le même choix et que l'on peut rejoindre ou qui permettent des économies d'échelle dont nous profiterons (Katz \& Shapiro, I985). Les langues produisent de telles externalités de réseau. La valeur instrumentale d'une langue, ceteris paribus, sera fonction du nombre d'individus qu'elle permet de rejoindre. Le choix d'apprendre ou non une langue, d'investir dans son apprentissage, de la transmettre, dépendra des bénéfices que l'on en attend, 
et ces bénéfices seront largement déterminés par les choix d'autrui d'apprendre ou de maintenir cette même langue. Ce qui motivera l'individu à intégrer un réseau se limite à l'utilité que le réseau de locuteurs potentiels est susceptible de lui procurer. Toutefois, en s'ajoutant au réseau, le nouvel agent fait davantage que simplement consommer un bien et en retirer un bénéfice: il en modifie la valeur à la hausse en offrant à chaque locuteur une nouvelle possibilité de communication. Cet ajout de valeur pour tous les membres du réseau n'influencera pas la décision de l'individu qui ne s'intéresse qu'à l'utilité que lui-même retirera de l'acquisition de l'appareil. L'utilité marginale que retire chacun de l'arrivée d'un nouveau locuteur est décroissante, mais l'utilité collective marginale produite par chaque nouveau locuteur est exponentielle. Il s'agit d'externalités puisque les bénéfices produits pour autrui lorsque l'on intègre un réseau ne seront pas pris en considération dans notre calcul coûts/bénéfices et n'influenceront pas notre décision.

\section{Des solutions aux échecs du marché linguistique}

Les interactions libres sur le marché linguistique créent donc des échecs de marché. En les reprenant, nous tâcherons d'identifier les interventions étatiques et les contraintes aux libertés linguistiques individuelles qui peuvent être légitimes afin de rétablir la coïncidence entre liberté et efficience, ou entre équilibre et optimalité. Ce que nous tenterons de démontrer, c'est que des individus rationnels, complètement libres dans leurs choix linguistiques, accepteraient certaines contraintes mutuelles à leurs comportements afin d'améliorer la situation de chacun. Nous sommes d'avis que ces contraintes doivent être aussi minimales que possible et sont justifiables dans la mesure où elles contribuent à la satisfaction des préférences individuelles et à l'élimination des problèmes d'action collective. En d'autres mots, respectant l'idéal des défenseurs du laisser-faire, les limites à la liberté ne doivent être justifiées que par une amélioration dans la capacité d'exercice de la liberté.

\section{L'asymétrie d'informations et les prophéties autocréatrices (ou autoréalisantes)}

Puisque la valeur pour un individu de faire partie d'un réseau est déterminée ceteris paribus par la taille de celui-ci, les croyances portant sur les éventuels choix linguistiques d'autrui influenceront largement les comportements linguistiques de chacun. Puisque les investissements nécessaires à l'apprentissage d'une langue sont relativement importants, et qu'ils sont relativement égaux pour toute langue, et puisque les bénéfices attendus sont radicalement différents selon que la langue choisie est largement partagée ou non, les individus auront une tendance à opter pour des «valeurs sûres». Plus une langue sera répandue, ou plus on s'attendra à ce qu'elle se répande, et plus d'individus réaliseront cette "prophétie autocréatrice ${ }^{1} »$ (Schelling, I978).

1. Chez Schelling: «self-fulfilling prophecy» (Schelling, I978: I I 5-I I9). 
L'idée générale, c'est que certaines attentes sont de nature à induire le type de comportement qui permettra la réalisation de l'attente en question. [...] Ce n'est bien évidemment pas la prophétie qui amène sa propre réalisation, mais plutôt l'attente, qui conduira par un enchaînement d'évènements et d'interactions à un résultat conforme à cette attente » (Schelling, I978: I I 5I 16) [Traduction libre].

L'attente d'un mouvement global vers l'anglais suffit à initier ce mouvement, tout comme l'attente d'une pénurie d'eau dans une ville donne lieu à cette pénurie. Les gens réagissent à leur environnement et aux changements anticipés dans cet environnement. En effet, le simple fait pour une communauté de croire qu'une langue se répandra suffit à ce qu'elle se répande en raison de réactions individuelles qui voient dans l'apprentissage de cette langue dominante en devenir un moyen d'améliorer leur situation. De la même façon, la simple croyance qu'une langue sera abandonnée massivement, que cette croyance soit fondée ou non, pourra motiver plusieurs parents à ne pas transmettre la langue et à précipiter cet abandon. Évidemment, la croyance que tous conserveront leur langue motivera à préserver celle-ci. Plus les investissements sont importants et plus l'aversion au risque joue un rôle important dans le choix des langues à apprendre. Les individus risquent donc souvent d'opter pour «le moindre mal d'une langue dominante ou une langue en voie de le devenir qui, à défaut de représenter une langue à laquelle ils s'identifient, représente un accès privilégié à une communauté linguistique importante et stable ». Pour prendre le risque de maintenir et de transmettre leur langue maternelle, ils devraient connaître les intentions des autres locuteurs qui les entourent. Or sans cette connaissance, ils doivent s'en remettre à des choix qui se fondent sur des informations faussées et empêchent la production de résultats optimaux.

Cette situation pose problème dans la mesure où les individus prendront des décisions qui, individuellement, leur offrent l'utilité attendue la plus importante. Le résultat suboptimal obtenu par la collectivité est causé par l'information imparfaite des préférences d'autrui. Sans connaître la valorisation relative qu'autrui donne aux fonctions identitaires et instrumentales des langues, et sans pouvoir profiter du système de prix, on ne peut connaître les préférences linguistiques d'autrui. Ce qui rend la situation encore plus problématique, c'est que chaque apprentissage fait à contrecœur représente dès lors une information sur les préférences d'autrui que celui-ci utilisera pour former ses propres préférences.

Dès lors, il est avantageux pour les individus de prendre une décision collective, de choisir un résultat auquel tous contribueront par leurs actions, plutôt que de choisir une stratégie individuelle susceptible de donner des résultats collectivement suboptimaux mais protégeant chacun contre la pire situation possible. Ils pourront, bien sûr, décider collectivement d'opter pour un transfert linguistique vers une langue dominante, de maintenir la langue vernaculaire, ou d'opter pour un répertoire linguistique particulier 
où chaque citoyen reçoit les moyens d'apprendre plus d'une langue. Ils prendront toutefois leur décision collectivement, consciemment, et non par dépit et en se fondant sur des croyances qui peuvent se révéler fausses ou incomplètes. Une stratégie collective est particulièrement adéquate dans le cas des langues en raison des externalités de réseaux, c'est-à-dire de la valeur de la langue largement déterminée par le nombre d'utilisateurs. Cette stratégie collective devra avoir préséance sur les stratégies individuelles et elle représentera donc une contrainte à la liberté linguistique de chacun. On pourra, par exemple, identifier une ou des langues officielles devant être parlées dans les institutions publiques et dans les milieux de travail. Cette contrainte n'est justifiée que par le fait qu'elle permet une satisfaction plus efficace des préférences.

\section{Internalisation des externalités}

Comme nous l'avons vu, les préférences linguistiques des individus sont multiples. Ils veulent d'une part maintenir la langue à laquelle ils s'identifient et dans laquelle ils excellent: généralement leur langue maternelle ou ancestrale. Ensuite, ils désirent communiquer par-delà les frontières de leur communauté linguistique et s'ouvrir au monde. Cette ouverture passe généralement par l'apprentissage d'une ou de plusieurs autres langues: les langues véhiculaires. Si les individus n'avaient que l'une ou l'autre de ces motivations et de ces préférences, des solutions simples s'offriraient à eux. Pour satisfaire la préférence de maintenir leur langue vernaculaire, une solution leur garantirait un résultat optimal: l'unilinguisme radical. Si le seul moyen de communiquer avec eux était de parler leur langue, et si les locuteurs étaient condamnés à naître, vivre et mourir dans la même communauté, la survie de la communauté linguistique serait garantie. De la même façon, si l'on observait que la préférence de maîtriser une langue véhiculaire, une autre option des plus efficace s'offrirait à eux: l'apprentissage de l'anglais par tous le plus rapidement possible. Maîtriser l'anglais permet d'être compris, de façon parfois incomplète et inexacte, mais à peu près n'importe où dans le monde.

Le problème se pose précisément parce que les individus ont des préférences multiples et qu'ils ne se satisfont pas de telles solutions simples et radicales. Les préférences pour les langues ne sauraient faire oublier les préférences dont la réalisation est rendue possible par les langues. C'est lorsqu'ils tentent de satisfaire ces multiples préférences exigeant la communication, lorsque les individus optent pour le cumul de deux ou plusieurs langues que leurs comportements risquent de créer des externalités et des problèmes d'action collective.

En effet, le bien collectif créé dans une communauté qui investit dans l'apprentissage d'une langue seconde commune pour la plupart de ses membres n'est pas simplement une langue, mais un répertoire linguistique permettant de maximiser les possibilités de communication efficace (De 
Swaan, 200I ; Laïtin, I992). Les compétences linguistiques d'une communauté, son répertoire linguistique, sont un bien collectif complexe. Or les individus ont des préférences qui associent les différentes langues à différents domaines ou rôles sociaux. Ils préféreront demeurer dans le confort chaleureux de leur langue maternelle aussi longtemps que possible et se hasarderont sur le territoire de la langue seconde lorsque cela leur sera nécessaire ou utile. Et telle est la situation optimale, telle est la situation souhaitée qui a motivé leur investissement dans l'acquisition d'une ou plusieurs langue(s) seconde(s). Une langue pour les «affaires internes ", qui est celle associée à l'identité et celle que l'on maîtrise le mieux, celle que l'on utilise pour s'exprimer précisément et finement sur des sujets importants, et une ou plusieurs langues pour les "affaires externes", utilisée(s) comme instrument lorsque la langue vernaculaire n'est pas (suffisamment) utile. Il semble que ce soit précisément dans ce bien que les individus ont investi. Ils n'investissent pas dans l'apprentissage de deux langues en n'ayant pas de préférence quant à l'utilisation qui sera faite de l'une et de l'autre. Ils n'investissent pas non plus nécessairement dans une langue seconde suivant une logique de bilinguisme soustractif où la nouvelle langue remplace la première. Ils investissent dans un répertoire linguistique où chacune des langues joue un rôle particulier et différent des autres, et où chacune est associée à des usages distincts. Ils désirent améliorer leurs possibilités de communication en apprenant une nouvelle langue, mais tout en conservant leurs acquis, c'est-à-dire leur langue et le contexte linguistique dans lequel ils excellent. C'est ici que le problème de resquillage et la production d'externalités négatives refont surface.

Lorsqu'une langue seconde est apprise massivement par les membres d'une communauté, cela crée des avantages importants pour les individus déjà locuteurs de cette langue nouvellement apprise. Ces individus peuvent en effet communiquer plus facilement avec les membres de la communauté, mais aussi venir s'y installer et espérer vivre en utilisant sa langue seconde. Par exemple, l'anglais est parlé par une proportion de plus en plus importante d'individus dans le monde entier. Dès lors, il est de moins en moins coûteux pour des anglophones, de langue maternelle ou seconde, de communiquer mais aussi de visiter des pays étrangers ou de s'y établir - du moins est-ce le cas dans les régions urbaines. Puisqu'il est de moins en moins coûteux de ne pas parler la langue vernaculaire de l'endroit où l'on s'établit une fois que l'on maîtrise l'anglais, et puisque l'apprentissage des langues secondes est motivé dans une large mesure par la nécessité, les incitatifs à apprendre les langues vernaculaires diminuent. Sans contrainte aux choix linguistiques des individus, le bilinguisme menace donc la stabilité de la situation linguistique souhaitée par les communautés investissant dans un répertoire linguistique particulier.

Le problème s'aggrave en raison des externalités de réseaux. En effet, la langue vernaculaire perd un peu de sa valeur chaque fois que la langue 
seconde la remplace dans les milieux de travail, dans les lieux sociaux, dans les écoles et les institutions. Et à l'inverse, la valeur de la langue véhiculaire sur le territoire s'en trouve augmentée. Ce sont là des externalités, dans la mesure où les individus qui n'apprennent pas la langue vernaculaire n'ont pas l'intention de nuire à la communauté linguistique locale. Ils désirent simplement communiquer aussi efficacement que possible, et à aussi bon marché que possible. Ces coûts n'influencent pas les comportements des individus non locuteurs des langues vernaculaires, mais ils sont néanmoins bien présents et assumés par les membres de la communauté linguistique vernaculaire.

La présence accrue de personnes ne maîtrisant pas la langue vernaculaire et se contentant de la langue véhiculaire pour mener leur vie imposera aussi d'autres types d'externalités négatives (Laponce, 2004). Plus le nombre d'individus incapables de s'exprimer et de comprendre la langue vernaculaire augmentera, plus on risquera d'être incapable d'établir un contact dans des situations plus ou moins graves et importantes. La capacité universelle de communication sur un territoire est un bien dont peuvent jouir les communautés linguistiques homogènes. Que ce soit pour demander une information banale, pour s'orienter ou pour appeler à l'aide, des bénéfices découlent de la compréhension généralisée.

Un autre type d'externalité négative est celle qui exerce une pression à la baisse sur les primes salariales pouvant être attendues par les individus qui investissent dans l'apprentissage de la langue seconde. Les bénéfices attendus à la suite de l'apprentissage d'une langue seconde par les locuteurs d'une communauté seront déterminés par deux facteurs: l'utilité de la compétence en question, et la difficulté à trouver quelqu'un possédant cette compétence à un niveau de perfectionnement donné. Dans le cas qui nous intéresse, la présence d'individus ne parlant que la langue véhiculaire, la langue seconde de la communauté considérée, fera augmenter le nombre d'individus ayant une compétence dans la langue en question. Ils auront donc tendance à faire réduire les bénéfices attendus de la connaissance de la langue seconde (Grin \& Vaillancourt). L'effet sera d'autant plus grand si ces individus ont la langue véhiculaire comme langue maternelle. Comme ils maîtriseront généralement mieux cette langue que les individus l'ayant comme langue seconde, ils réduiront les bénéfices attendus de l'apprentissage de cette langue comme langue seconde. Il y a donc présence d'externalités négatives, le comportement de certains individus influençant à la baisse la valeur des compétences linguistiques d'autres individus.

À ce tableau, nous pouvons ajouter une autre intervention de l'État qui se justifie par la nécessité d'internaliser les externalités. Church et King (I993) ont démontré qu'en raison des externalités de réseaux produites par l'apprentissage d'une seconde langue dans une population il est possible que les individus n'aient pas les incitatifs suffisants pour les motiver à l'apprentissage. Si la décision de ceux-ci n'est fondée que sur les bénéfices qu'ils 
peuvent personnellement attendre, et si l'on néglige les bénéfices produits pour tous les locuteurs parlant aussi cette langue seconde, il risque d'y avoir moins d'apprentissage linguistique que ce qui est collectivement optimal. Dès lors, une intervention de l'État subventionnant l'apprentissage de la langue est jugée légitime sur la base d'arguments d'efficacité prenant en considération la présence d'externalités de réseaux.

\section{Une réponse à une critique et deux prises de distance}

Nous devons maintenant répondre à une critique et prendre nos distances par rapport à deux autres utilisations du concept de resquillage linguistique. Nous affirmons qu'il y a bel et bien resquillage de la part des individus refusant d'apprendre la langue vernaculaire puisqu'ils abusent du bien collectif créé par la communauté locale en l'utilisant d'une façon nuisible à cette dernière. Si le bien créé était une seule langue, Weinstock aurait raison d'avancer que le resquillage est impossible dans la mesure où personne ne peut profiter des bénéfices d'une langue sans investir dans son apprentissage et contribuer à son maintien (Weinstock, 2003). On ne pourrait avancer que les membres de la communauté linguistique menacée resquillent lorsqu'ils apprennent une langue dominante en laissant les autres membres de leur communauté assumer les coûts du soutien d'une langue puisqu'il est possible qu'ils ne la valorisent plus. Comme nous l'avons présenté, les langues ont une valeur identitaire et une valeur instrumentale, et les locuteurs peuvent valoriser différentes langues de diverses manières selon ces deux dimensions; il est donc impossible d'affirmer qu'il y a resquillage sur la création d'une seule langue. Il n'y a donc pas resquillage sur le maintien de la langue vernaculaire comprise comme bien collectif. Il y a resquillage dans la mesure où des individus utilisent les compétences linguistiques de la communauté en question d'une façon qui réduit la valeur de leur bien linguistique et réduit le retour attendu sur leur investissement linguistique. Ils profitent de la situation créée par les compétences linguistiques de la communauté d'une façon qui n'est pas souhaitable pour celle-ci. Notons que, dans ce tableau, chaque individu agit rationnellement. Le problème, c'est que plusieurs communautés linguistiques minoritaires seront menacées si nous laissons les individus agir d'une façon rationnelle individuellement, mais problématique collectivement.

Cette position est différente des deux propositions qui sont offertes par Philippe Van Parijs et Idil Boran. Pour Van Parijs, on peut critiquer les individus ayant la lingua franca comme langue maternelle et qui refusent d'investir dans l'enseignement de leur langue pour d'autres locuteurs afin d'en faire la lingua franca d'une région ou du monde (Van Parijs, 20 I I). Or dans une logique de justice coopérative, pour recourir à l'accusation de resquillage, il faudrait démontrer que la participation et la contribution des anglophones sont nécessaires pour rendre rationnel l'apprentissage de l'anglais par les non-anglophones, et que la création d'un bien collectif tel que 
l'anglais comme lingua franca est désirée par tous. Ici, il semble que la participation des anglophones ne soit pas nécessaire pour que la création du bien en question soit désirable.

Une autre proposition vise à critiquer l'absence de contribution à la diversité linguistique, laquelle est un bien que tous apprécient et dont tous profitent (Boran, 2003: 200-20I). Le bien collectif est ici la diversité linguistique, et non une langue en particulier. Les locuteurs de langues dominantes menacent la diversité linguistique, en plus de ne pas y contribuer. Le problème de cette position est qu'il ne va pas de soi que tous apprécient et profitent de la diversité linguistique davantage qu'ils n'apprécieraient l'unilinguisme global. De plus, considérer que les locuteurs de langues dominantes ne contribuent pas à la diversité linguistique semble difficile à accepter puisque c'est précisément de la diversité des langues dominantes que nous retirons le plus de bénéfices au quotidien. Beaucoup de gens se sont offert par l'apprentissage d'une langue dominante étrangère un accès privilégié à des auteurs et à des œuvres, à des gens et à des expériences auxquels ils n'auraient pas eu accès autrement. On peut accepter que toutes les langues renferment autant de beauté et de richesses, qu'elles sont autant d'accès à l'esprit humain et qu'elles dévoilent des connaissances particulières, mais il demeure que la très vaste majorité d'entre nous n'entrerons en contact et ne profiterons de la diversité linguistique qu'à travers une dizaine de langues - dominantes pour la plupart d'entre elles. À moins de qualifier la diversité linguistique souhaitée, toute langue contribue à la diversité. Ce qui est apprécié, c'est une certaine diversité linguistique, pas cette diversité linguistique particulière. On ne peut donc accuser de resquillage quiconque agit de façon à ne pas contribuer à la diversité linguistique actuelle sans avoir démontré qu'il profite effectivement de la diversité telle que nous la connaissons.

\section{Échecs de marché et interventions de l'État}

Les interventions de l'État qui pourront être justifiées viseront à internaliser certains coûts imposés à des communautés linguistiques ayant investi dans une langue seconde, à faire en sorte que les bénéfices de l'apprentissage reviennent aux membres de la communauté, et que les coûts imposés par les non-locuteurs de la langue vernaculaire soient aussi faibles que possible. L'internalisation de ces externalités est nécessaire afin de rétablir une certaine coïncidence entre l'équilibre des choix individuels et l'optimalité des résultats collectifs. C'est la recherche de cette coïncidence qui nous oblige à quitter le libre marché linguistique et qui rend inefficace la politique de laisser-faire dans le domaine linguistique. Les individus, au nom de leur liberté linguistique, préféreront être contraints dans certains de leurs choix linguistiques plutôt que d'être entièrement libres et que des problèmes d'action collective viennent miner leurs efforts de maximisation d'utilité.

On pourrait avancer que l'élimination de ces externalités pourrait se faire sur une base individuelle et sans intervention ou contraintes de l'État. 
En effet, si chacun s'imposait une autocontrainte faisant en sorte de ne parler que la langue vernaculaire dans les communications internes, le problème serait en partie réglé. Les individus garderaient pour eux leur avantage et n'utiliseraient leur langue seconde que lorsque cela est à leur avantage. Mais cette solution pose un problème d'action collective. Les intérêts individuels et les intérêts collectifs divergent. Par exemple, les gens semblent trop respectueux des normes de civilité pour refuser de répondre à quelqu'un qui les interroge dans leur langue seconde ${ }^{2}$. En fait, les individus ont davantage intérêt à répondre. Ils font une bonne action, pratiquent leur compétence linguistique, s'évitent des remontrances ou des injures, et il est assez peu probable que ce soit leur comportement qui sonne le glas de la langue vernaculaire. Les individus semblent trop gentils pour protéger efficacement leur langue. Les externalités négatives produites par ces individus sont modestes. Cependant, étendons un tel comportement à des communautés entières, et il devient menaçant pour la stabilité de plusieurs langues vernaculaires.

Devant ce tableau, si les individus désirent maintenir en santé leur langue vernaculaire et profiter des avantages offerts par la lingua franca qu'est l'anglais (ou toute autre langue régionale dominante), ils devront s'imposer des contraintes quant à la langue (ou aux langues) pouvant être utilisée(s) dans certains contextes. Les contraintes peuvent s'appliquer à un territoire, à un rôle ou à une période de temps donnés (Laponce, I984). Il peut s'agir de lieux plus ou moins étendus, comme les institutions d'une province ou d'une ville, ou de périodes de temps, telles les réunions professionnelles internes. Ces contraintes sont nécessaires afin d'éviter la production d'externalités négatives excessives et éliminer le resquillage sur les compétences linguistiques de la communauté. Si les individus membres d'une communauté linguistique apprennent une langue seconde pour améliorer leur situation, il est probable qu'ils refuseront que cet apprentissage devienne la porte d'entrée de la plus importante menace à leur langue vernaculaire. Il est donc rationnel pour chaque individu de refuser de subir les coûts externes imposés par les choix d'autrui, et d'imposer certaines restrictions linguistiques sur un territoire donné. Ils contrôlent de cette façon les externalités positives qu'ils créent en apprenant massivement la langue seconde ainsi que les externalités négatives pouvant leur être imposées sur le territoire.

Ronald Coase a bien mis en lumière le fait que la relation de nuisance dans les relations impliquant des externalités est réciproque et non unilatérale (Coase, 2005). Empêcher $x$ d'imposer des externalités à $y$ impose des coûts à $x$ alors que le lui permettre impose évidemment des coûts à $y$. Si des politiques linguistiques peuvent nuire aux locuteurs d'autres langues et leur

2. Nous croyons que, même armés de lois pour appuyer leurs gestes, les individus agiraient en respectant les normes du civisme, seraient trop gentils pour protéger efficacement leur langue. Notre intuition s'oppose sur ce point précis à l'hypothèse de Van Parijs (20I I). 
imposer des coûts, la présence de ces mêmes locuteurs et les choix linguistiques qu'ils font peuvent aussi nuire aux populations locales et leur imposer des coûts importants. La question de savoir si une politique linguistique impose des coûts aux communautés linguistiques non protégées n'est donc pas celle qui doit nous intéresser ici. La réponse est évidente et affirmative. On doit plutôt se demander comment trancher de façon raisonnable et déterminer quels coûts peuvent légitimement être imposés par les actions et les choix linguistiques des individus. En d'autres mots, le simple fait d'identifier les coûts imposés à un individu, la limitation à sa liberté, ne suffit pas à condamner la politique linguistique au nom de la liberté individuelle. Nous devons comparer les coûts imposés dans les deux scénarios possibles et trancher en faveur d'un de ces scénarios.

Nous pouvons tirer un autre enseignement de l'œuvre de Coase en ce qui a trait à la réaction institutionnelle que l'on peut avoir face à la présence d'externalités. Nous devons comprendre que l'optimalité n'exige pas d'éliminer complètement les externalités. Il est possible en effet qu'une situation soit plus désirable, même s'il y a présence d'externalités. L'important est que la situation soit préférable pour tous. L'élimination de toute communication interne dans une langue autre que la langue vernaculaire ne sera pas souhaitable. L'idée, c'est de faire en sorte qu'il soit incontournable d'apprendre la langue vernaculaire pour vivre une vie riche et bien remplie sur un territoire donné. Mais pas en sacrifiant les avantages que procure la connaissance de la langue véhiculaire (tourisme, affaires, etc.). Chaque communauté pourra donc identifier le domaine de chacune des langues composant son répertoire linguistique et le type d'interventions étatiques justifiables dépendra des préférences des locuteurs dans chaque situation.

\section{Conclusion}

Comme nous avons tenté de l'expliquer, la liberté sans contrainte exige, pour donner des résultats optimaux, des conditions qui ne sont pas rencontrées dans le cas des langues et du marché linguistique. Dès lors, on ne peut laisser les individus faire des choix linguistiques et espérer des résultats optimaux. Ce qui est à prédire, c'est que les choix en équilibre des individus produiront des résultats qui ne sont pas désirés par la plupart d'entre eux. Cela est d'autant plus vrai lorsque l'on considère la présence d'autres types d'échecs de marché comme l'absence de marchés (l'absence de préférence des générations futures sur le marché) et la présence de biens collectifs sur le marché (les langues étant des biens collectifs). Cette suboptimalité s'incarne entre autres dans la pression mise sur la très vaste majorité des 6000 langues parlées sur la planète. À part quelques communautés très dominantes qui ne sont pas menacées directement par d'autres langues, les communautés linguistiques font face à une réelle menace. Notre proposition permet donc de justifier, pour les individus, une liberté linguistique maximale compatible avec l'atteinte de résultats optimaux. La solution passe par une certaine 
autonomie linguistique des communautés et une séparation territoriale nécessaire à la protection de leur langue si tel est leur désir. Puisque, selon nous, toutes les communautés devraient avoir cette liberté, il existe une réciprocité entre les individus et entre les communautés dans l'imposition de coûts liée aux restrictions linguistiques. Lorsque présent sur le territoire de l'autre, chacun devra assumer les mêmes coûts. En contrepartie, chacun, sur son propre territoire, profitera au maximum du bien collectif qu'il a contribué à produire et sera lui-même garant de sa stabilité. La situation linguistique sera ainsi plus stable et moins incertaine. Il est aussi important de rappeler que ces coûts imposés de l'intérieur par l'intervention d'institutions politiques et légales ne sont pas imposés unilatéralement mais visent à réagir face à l'imposition de coûts imposés de l'extérieur. Puisque certains coûts seront nécessairement imposés, il semble intuitivement acceptable que ceux qui les assument soient ceux qui les créent en faisant le choix de se déplacer et de quitter leur territoire linguistique. Ce sera à eux d'assumer les coûts imposés par la diversité linguistique dans un monde en rétrécissement constant, et non à ceux qui ont fait le choix de demeurer sur leur territoire.

Nous pouvons justifier l'intervention étatique sur le terrain de l'économie et sur celui de la morale. La création de coûts externes pose d'abord un problème économique, lequel empêche que les choix individuels produisent des résultats optimaux. C'est donc sur la base d'une amélioration de l'efficacité du processus de choix linguistiques que l'on peut justifier l'intervention étatique. Mais du point de vue de la morale, ce n'est pas l'inefficacité causée par les coûts externes mais l'imposition de ces coûts à autrui sans son aval qui pose problème. Nul ne saurait légitimement imposer les coûts de ses choix à autrui sans son approbation. Or la production de coûts externes implique exactement un tel transfert illégitime de coûts. On peut donc souhaiter la création d'institutions régulant les choix individuels de façon à ce que les résultats soient optimaux, ou de façon à ce que personne n'ait à assumer les coûts des comportements et des choix d'autrui.

L'imposition de limites aux choix linguistiques est tout à fait acceptable pour quiconque est conscient de la réalité linguistique actuelle, des menaces qui pèsent sur les langues minoritaires ou sur leurs locuteurs et désire conserver sa langue maternelle tout en profitant d'une lingua franca. La reconnaissance du fait que les libres choix non contraints donnent des résultats indésirables semble suffisante pour justifier l'imposition de contraintes permettant à chacun de mieux satisfaire ses préférences linguistiques, et ce, même à l'intérieur d'un cadre théorique où la liberté individuelle prime.

\section{Bibliographie}

Barro, R. J. Getting it Right. Cambridge, MIT Press, I996.

Boran, Idil. "Global Linguistic Justice, Public Goods, and the Principle of Fairness ", Language Rights and Political Theory, A. Patten \& W. Kymlicka (eds.), Oxford University Press, 2003, p. I89-209. 
Carr, Jack. «Le bilinguisme au Canada: L'usage consacre-t-il l'anglais monopole naturel ? ", Économie et langue, François Vaillancourt (dir.), Éditeur officiel du Québec, 1985, p. 27-38.

Church, Jeffrey \& Ian King. "Bilingualism and Network Externalities ", The Canadian Journal of Economics, Vol. 26, No. 2, May 1993, p. 337-345.

Coase, Ronald H. «Le problème du coût social ", L'entreprise, le marché et le droit, Paris, Les éditions d'Organisation, 2005, p. I I7-I 80.

Coulombe, Pierre A. Language Rights in French Canada, New York, Peter Lang Publishing, I995, I 83 p.

Dalmazzone, Silvana. "L'économie de la langue: méthode des externalités de réseau ", Explorer l'économie linguistique, Albert Breton (dir.), série Nouvelles perspectives canadiennes, Patrimoine canadien, ministère des Travaux publics et des Services gouvernementaux du Canada, Ottawa, I 999, p. 69-94.

De Swaan, Abram. Words of the World, Cambridge, UK, Polity Press, 2001, 272 p. Friedman, Milton. Capitalisme et liberté, Paris, Les éditions Leducs, 2010, 3 I 6 p.

Gauthier, David. Morale et contrat, Sprimont, Mardaga, 2000, 427 p.

Grin, François. "Economic Considerations in Language Policy », An Introduction to Language Policy. Theory and Method, Malden, MA, Blackwell Publishing, 2006, p. 77-94.

Grin, François \& François Vaillancourt. «The Economics of Multilingualism: Overview and Analytical Framework", Annual Review of Applied Linguistics, Vol. I7, I997, p. 43-65.

Katz, Michael L. et Carl Shapiro. "Network Externalities, Competition, and Compatibility", The American Economic Review, Vol. 75, No. 3, June I98 5, p. 424-440.

Kukathas, Chandran. "Liberalism and Multiculturalism: The Politics of Indifference", Political Theory, vol. 26, no. 5, Oct. I998, p. 686-699.

Kukathas, Chandran. "Are There Any Cultural Rights?", Political Theory, Vol. 20, No. I, Feb. I992, p. I05-I39.

Laitin, David D. Language Repertoires and State Construction in Africa, Cambridge, Cambridge University Press, I992, 205 p.

Laponce, Jean. "Comments on Laitin and Grin", Cultural Diversity Versus Economic Solidarity, Philippe Van Parijs (dir.), Bruxelles, Les éditions De Boeck Université, 2004, p. 203-209.

Laponce, Jean. Langue et territoire, Québec, Presses de l'Université Laval, I984, 265 p.

Lipsey, R. G. \& Kelvin Lancaster. "The General Theory of Second Best ", The Review of Economic Studies, Vol. 24, No. I, I956 -I957, p. I I-32.

Rawls, John. "Fairness to Goodness ", The Philosophical Review, Vol. 84, No. 4, Oct. I975, p. 536-554.

Réaume, Denise. "Official-Language Rights: Intrinsic Value and the Protection of Difference", Citizenship in Diverse Societies, Will Kymlicka \& Wayne Norman (eds.), Oxford University Press, 2000, p. 245-272.

Robichaud, David \& Helder De Schutter. «Language is Just a Tool! On the Instrumentalist Approach to Language ", Cambridge Handbook on Language Policy, Bernard Spolsky (ed.), Cambridge University Press (à paraître).

Robichaud, David \& Patrick Turmel. «La transmission intergénérationnelle du patrimoine: un problème de justice ", Générations équitables, Dan Sylvain et Jeorg Tremmel (dir.), coll. Sciences humaines et sociales, Paris, Éditions Connaissances et Savoirs, 20I0, p. 22 I-238. 
Schelling, Thomas C. Micromotives and Macrobehavior, New York, Norton and Company, 1978, $272 \mathrm{p}$.

Van Parijs, Philippe. Linguistic Justice for Europe and for the World, Oxford, Oxford University Press, 20I I, 299 p.

Weinstock, Daniel. "Le paradoxe des droits linguistiques", dans Communauté de droits, Droit des communautés, Ysolde Gendreau (dir.), Montréal, Éditions Thémis, 2003, 43-83.

- - "The Antinomy of Language Rights", Language Rights and Political Theory, A. Patten \& W. Kymlicka (eds.), Oxford University Press, 2003a, p. 250-270. 